\title{
HLA-Derived Peptides as Novel Immunosuppressives
}

\author{
ALAN M. KRENSKY \\ Stanford University School of Medicine, Stanford, California 94305-5119
}

\begin{abstract}
Peptides corresponding to linear sequences of HLA molecules have been synthesized and tested for immunomodulatory activity in in vitro assays using human $\mathrm{T}$ lymphocytes. Sequences from different parts of the HLA molecules have different effects. Peptides corresponding to residues 75-84 of an HLA class I supratypic specificity of limited heterogeneity (HLABw4) had profound inhibitory effects in a variety of in vitro

flux and interrupting normal signal transduction pathways. Furthermore, these peptides bind to members of the heat-shock protein 70 family, implicating these ubiquitous proteins in the immunomodulatory pathway. Such peptides may be normal physiologic mediators. In any case, they represent potential new immunotherapeutics for a variety of immune-mediated diseases. (Pediatr Res 38: 275-279, 1995)
\end{abstract} assays of human $T$ lymphocyte function. Furthermore, a 2-wk course of human HLA sequences and cyclosporine therapy induced enduring immunologic tolerance in a rat model of heterotopic heart transplantation. These studies prompted clinical trials which are currently in progress. The peptides appear to induce $T$ cell anergy by causing a prolonged intracellular calcium

The function of the immune system is the discrimination of self from non-self. As organisms evolved from single cells to multicellular, it became imperative to recognize and destroy non-self. Foreign invaders (infectious organisms) and dysregulated self (tumors) must be removed to ensure the integrity of the whole animal. Man has evolved a complex, apparently redundant, and highly effective system to perform this function. Its normal operation is part of everyday life, but its abnormal function can lead to human disease. The premise of work in my laboratory is that, by understanding the basic biologic mechanisms of the immune response, we can design novel immunotherapies for use in a myriad of disease states. We have concentrated our efforts on understanding T lymphocyte function because these cells are pivotal regulators and effectors in a variety of disease states. In some cases, including transplant rejection, autoimmunity, and allergy, the immune response is too strong and must be down-regulated. In other instances, including infection, immunodeficiency, and cancer, the immune response is inadequate and must be up-regulated. Immunomodulation in either direction should be possible if we

Correspondence and reprint requests: Alan M. Krensky, M.D., Shelagh Galligan Professor of Pediatrics, Stanford University School of Medicine, Stanford, CA 943055119.

Dr. Krensky is a recipient of the Society for Pediatric Research 1995 E. Mead Johnson Award for Research in Pediatrics.

\author{
Abbreviations \\ CTL, cytotoxic T lymphocyte \\ hsp, heat-shock protein \\ NF-AT, nuclear factor of activated T cells
}

understand the fundamental mechanisms involved in antigen recognition, activation, differentiation, and effector function by T lymphocytes.

The basic mechanisms underlying $\mathrm{T}$ cell biology have been recently detailed elsewhere $(1,2)$. T lymphocytes recognize antigen as peptides in the context of self HLA molecules. Helper $\mathrm{T}$ lymphocytes generally express the CD4 cell surface molecule and recognize antigen in the context of HLA class II molecules (such as HLA-DR). The $\alpha, \beta \mathrm{T}$ cell receptor heterodimer on their surface binds peptide in the context of self HLA. Once triggered via antigen-specific recognition, the $\mathrm{T}$ cell receptor sends signals through associated polypeptides of the CD3 complex, which in turn, result in a series of biochemical events near the cell membrane. Calcium increases within the cell from both extracellular and intracellular sources. An inositol phosphate pathway is activated. Protein kinase $\mathrm{C}$ and tyrosine kinases are activated, resulting in a series of new phosphorylations. Together these events lead to the activation of a cascade of molecular interactions which eventually result in new gene expression. Phosphorylation of cytoplasmic proteins lead to the nuclear translocation and binding of transacting DNA binding proteins to cis-acting sequences of genes. These regulatory proteins control gene transcription. Messenger RNA is transcribed and proteins translated. IL-2, for example, is made in response to $\mathrm{T}$ cell activation. This impor- 
tant growth and differentiation factor results in both autocrine (self) and paracrine (nearby) $\mathrm{T}$ cell stimulation. Such a basic understanding of the events involved in $T$ cell activation indicates several potential sites at which to interrupt or augment the process (Table 1). Although we and others have investigated all of these steps in the activation process, the remainder of this review will deal exclusively with the first target of opportunity, i.e. the HLA-peptide interaction.

\section{HLA DERIVED PEPTIDES}

CTL were used to define the important structures of HLA molecules involved in allorecognition $(3,4)$. Synthetic peptides corresponding to CTL target sequences present in the HLA molecule were made and tested in CTL assays to see if they would inhibit or augment cytotoxicity $(5,6)$. Interest first turned to a region in the $\alpha_{2}$ domain of HLA-A2 (Fig. 1). Our collaborator, Peter Parham (Stanford), had shown that a MAb, PA2.1, recognized residue 107 of HLA-A2 and that the amino acid substitution of glycine (in all other HLA types) for tryptophan (in HLA-A2) accounted for the HLA-A2 specificity of the PA2.1 MAb $(5,7)$. We found that this single amino acid difference also accounted for the immunodominant epitope recognized by most allogeneic CTL $(4,5)$. Site-directed mutagenesis of residue 107 from tryptophan to glycine resulted in a loss of most cell-mediated cytotoxicity directed at HLA-A2 (4). These observations led us to test the effect of a synthetic peptide corresponding to this target sequence on in vitro cytotoxicity (5). We found that a peptide corresponding to residues 101-108 of HLA-A2 profoundly inhibited HLA-A2directed cytotoxicity, whereas a control peptide, corresponding to residues 101-108 of the closely related allele, HLA-Aw68, had no effect. Furthermore, this inhibition was allele-specific. The HLA-A2-derived peptide would block only HLA-A2directed CTL. We were able to generalize this finding to other epitopes of HLA-A2 and other alleles $(6,8)$. In general, a peptide corresponding to the putative target sequence would inhibit allele specific recognition. Although this finding has significant biologic implications, indicating that synthetic peptides can mimic target structures and block cytotoxicity by competition, this approach was limited in its clinical utility. A very large mixture of peptides would be required to cover all of the potential epitopes and alleles involved in any specific donor-recipient pair. Therefore, our interest turned to allele nonspecific interactions.

Table 1. Potential sites at which to interrupt $T$ lymphocyte-mediated transplant rejection $(1,2)$

\footnotetext{
1. HLA-peptide interactions

2. T cell receptor (TCR) recognition of HLA plus peptide

3. CD3-TCR complex mediated signal transduction

a. Calcium flux

b. Phosphatidyl inositol pathway

c. Protein kinase $\mathrm{C}$

d. Tyrosine kinase

4. Other ccllular adhesion and accessory signal transduction pathways

5. Second messengers

6. DNA binding regulatory proteins and cis-acting binding sites

7. Effector molecules (perforins, granzymes, etc.)
}

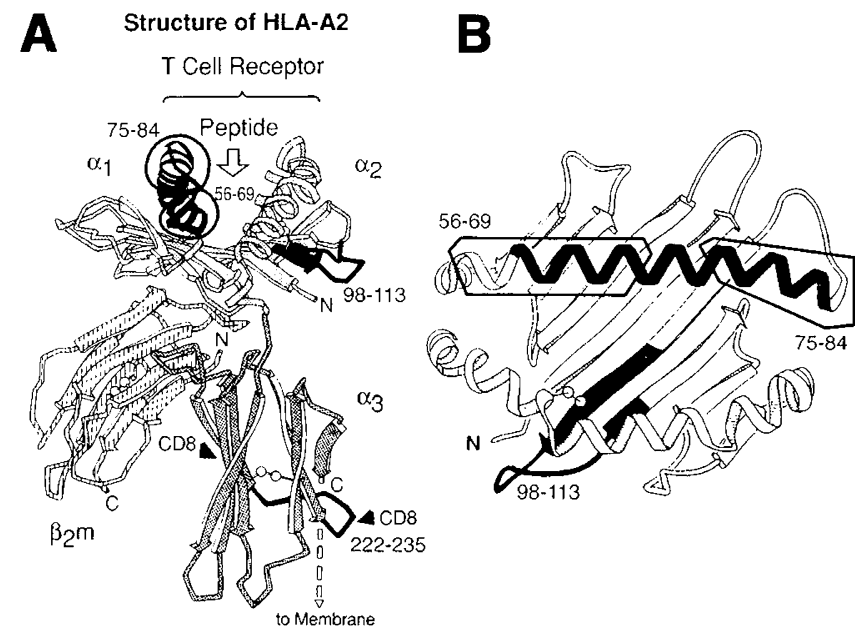

Figure 1. Model of the crystal structure of an HLA class I molecule (HLA$A 2$ ), showing the location of the sequences corresponding to the various immunomodulatory peptides: $(A)$ side view, $(B)$ top view. Synthetic peptides corresponding to residues $56-69,60-84,98-113$, and 222-235 can inhibit T cell function. Potent allele nonspecific inhibition is seen with sequences corresponding to residues 75-84 of HLA-B2702 and -B38, corresponding to the HLA-Bw4a supratypic specificity $(3,14,16)$.

\section{THE CD8 BINDING LOOP OF THE HLA CLASS I $\mathrm{A}_{3}$ DOMAIN}

With Dan Littman (UCSF) and Peter Parham (Stanford), we helped to define a loop of the HLA class I $\alpha_{3}$ domain which appeared to be involved in binding to the CD8 co-receptor on $\mathrm{T}$ cells $(9,10)$ (Fig. 1). We prepared a synthetic peptide corresponding to this charged and exposed loop and tested it for inhibition of cytotoxicity (11). Because CD8 appeared to be required for cytotoxicity and anti-CD8 MAb-inhibited cytotoxicity (12), we reasoned that peptides corresponding to this loop may competitively inhibit the CD8-HLA interaction and result in inhibition of killing. Although such peptides efficiently inhibited the differentiation of CTL from precursors (measured by limiting dilution analysis), they did not reproducibly inhibit killing by fully differentiated CTL (11). In retrospect, this result may not be surprising in that the interaction of CD8 and HLA appears to be most pivotol in the earliest stages of $\mathrm{T}$ cell recognition and activation (13). Fully differentiated CTL have high affinity for their targets and are generally less easily inhibited by anti-CD8 MAb (9).

\section{PEPTIDES CORRESPONDING TO THE HLA CLASS I A 1 A-HELIX}

Thus, the goal of identifying an allele nonspecific, yet broad and effective, peptide inhibitor of cytotoxicity had not yet been reached. We next turned our interest to the $\alpha$-helices (Fig. 1) (14). The $\alpha$-helices of HLA class I appeared to interact with both the target peptides present in the antigen-presenting groove and the $\mathrm{T}$ cell receptor above (15). Reasoning that peptides corresponding to these sequences might competitively inhibit important contact regions between antigenic peptide, HLA, and T cell receptor, we synthesized peptides corresponding to sequences of the $\alpha_{1} \alpha$-helix of nine different HLA class I alleles $(14,16)$ (Table 2$)$. The $\alpha$-helical peptides showed a 
Table 2. Sequences of HLA peptides corresponding to $\alpha_{1} \alpha$-helical sequences of HLA class I molecules

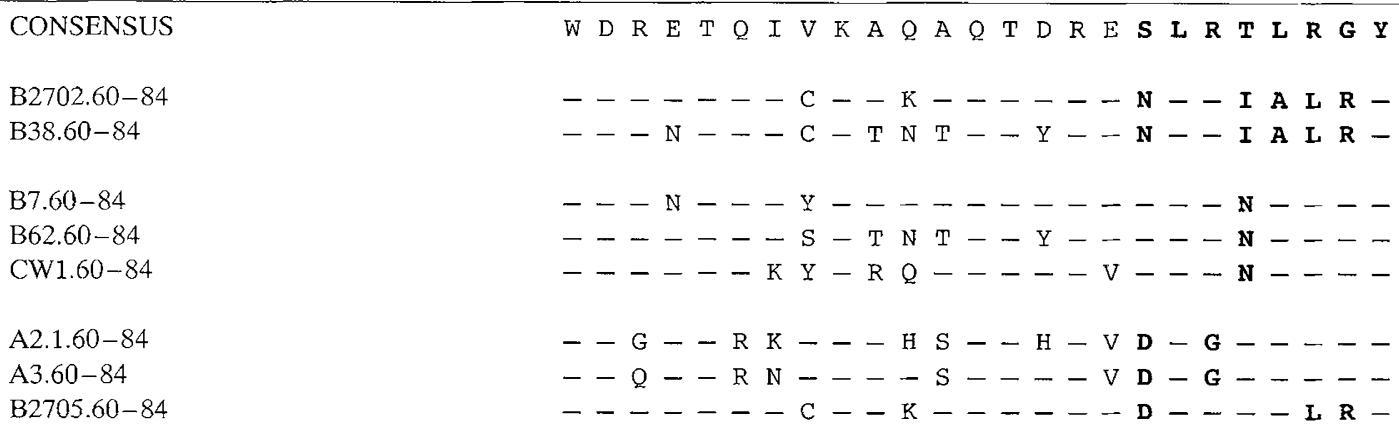

Peptides corresponding to the first group (HLA-B2702 and -B38) inhibit the generation of CTL and cytolysis by established CTL. Peptides corresponding to the second group (HLA-B7, -B62, and -CW1) inhibit only the generation of CTL. Other sequences (HLA-A2, -A3, and -B2705) have no effect. These functional effects correlate with the sequences shown in bold $(14,16)$.

variety of effects. Some inhibited cytotoxicity and other in vitro assays. Others inhibited only the differentiation of CTL in limiting dilution analysis, reminiscent of the activity of peptides corresponding to the CD8 binding loop. Still others had no effect on functional assays in vitro. Strikingly, these different effects were entirely attributable to the linear amino acid sequence of the carboxy peptides of the $\alpha_{1} \alpha$-helix (Table 2) $(14,16)$.

These profound inhibitory effects in vitro provided the impetus to test the peptides as immune modulators in vivo. Unexpectedly, the peptides corresponding to human sequences also inhibited in vitro assays in other species, including monkeys, rats, and mice. We used a rat heterotopic heart transplant model to test the efficacy of the peptides in vivo. In a report by Nisco et al. (17), as little as five doses of cyclosporine and four doses of peptide resulted in indefinite graft survival in $80 \%$ of animals (Fig. 2). In addition, these animals were tolerant to their grafts. They were capable of rejecting a third party graft but would accept a subsequent graft from the same donor. This tolerance was not tissue-specific in that a subsequent skin graft was tolerated after a heart transplant. Mechanistically, this tolerance was due to anergy, not clonal deletion or suppression. Donor-reactive $\mathrm{T}$ cells were in fact present, but they were unable to function in transplant rejection. Perhaps most surprising was the fact that the peptides worked just as well when given orally as intravenously (17).

\section{TOLERANCE INDUCTION AND MAINTENANCE}

Tolerance is the "holy grail" of transplantation biology (18, 19). The ability to induce and maintain antigen-specific nonresponsiveness without generalized immunosuppression portends an era when patients no longer require chronic drug therapy with the concomitant side effects and risks of infection and secondary tumors. Rather, tolerance denotes a true cure for end organ failure. A foreign graft is no longer recognized as non-self. The HLA peptides described above produce and maintain this effect in animal models. These findings gave rise to clinical trials of peptides in people. A phase I trial indicated that the peptides were nontoxic for use in humans. SangStat Medical Corporation, Menlo Park, CA, is currently conducting a phase II trial of these peptides under the brand name "Allotrap."

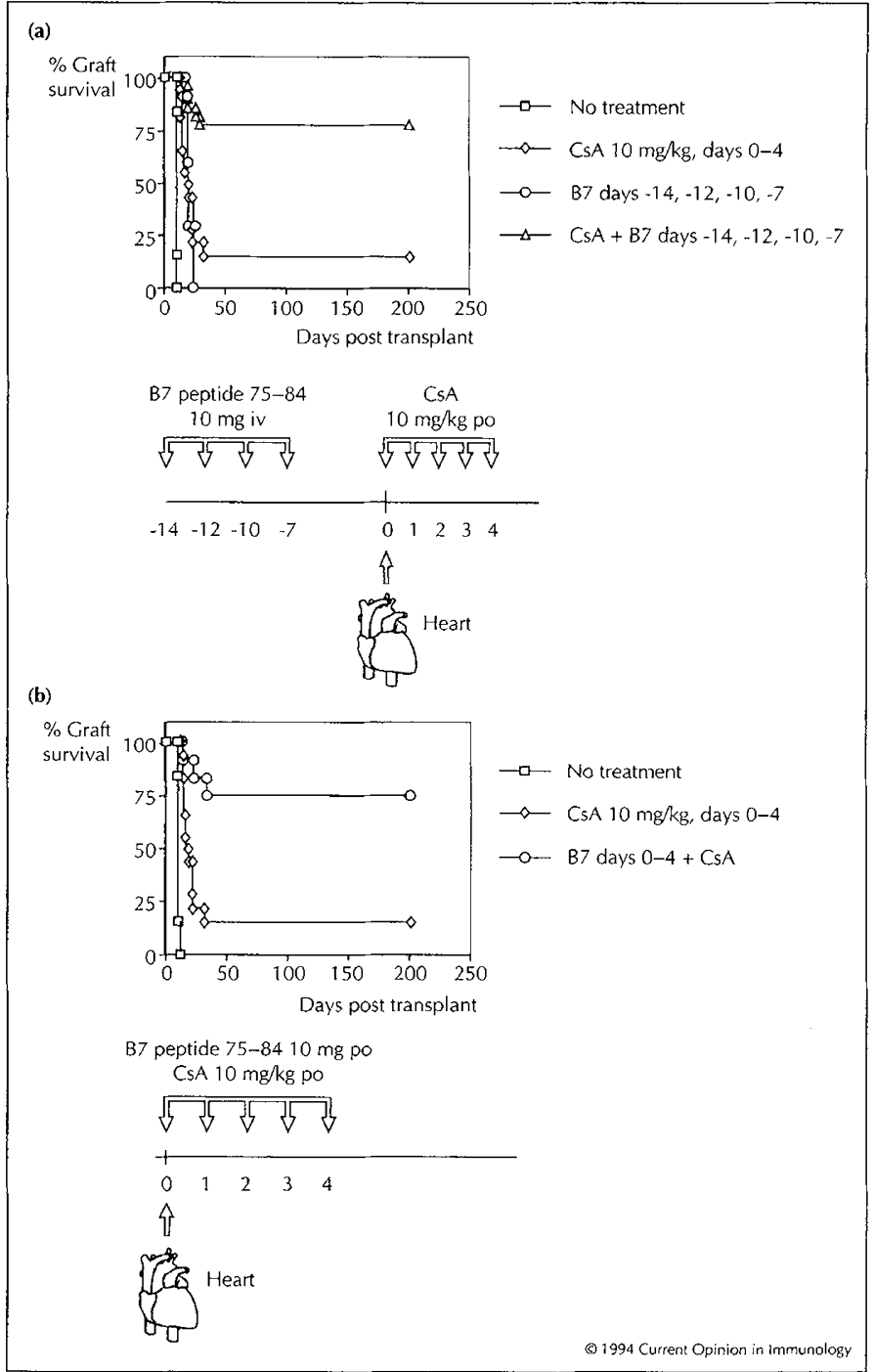

Figure 2. Survival of rat heterotopic heart allografts. ACI rats were recipients of abdominal heterotopic cardiac allografts from LEW donors. Grafts were palpated daily and deemed rejected when no beat was detected. A synthetic peptide corresponding to residues 75-84 of HLA-B7 (denoted B7) was administered by either: $(A)$ intravenous injection or $(B)$ orally by gavage. These data are adapted from Nisco et al. and reprinted with permission from Current Opinion in Immunology (25). 


\section{MECHANISM OF ACTION OF PEPTIDES CORRESPONDING TO RESIDUES 75-84 OF HLA-BW4}

While the clinical trials are ongoing, interest in our laboratory has turned to the mechanism of action of these peptides. One direction involves investigation of the effects of the peptides on events required for $T$ cell activation and differentiation. A second approach involves identification and characterization of the $\mathrm{T}$ cell receptor for the immunomodulatory peptides.

HLA-derived peptides cause a calcium flux in T lymphocytes. Early events after $\mathrm{T}$ cell activation include the flux of intracellular calcium from both internal and external sources (1). Antibodies against the $T$ cell receptor complex and antigen cause a marked increase in intracellular calcium in $T$ cells, measured by $\mathrm{Ca}^{2+}$ binding dyes, such as Indo-1 (Fig. 3) (20). Using such approaches, we have shown that the immunomodulatory HLA-derived peptides described above cause sharp increases in intracellular calcium (16). Furthermore, this flux is prolonged, lasting longer than $30 \mathrm{~min}$. It is likely that the rise in intracellular calcium causes T cell anergy. Jenkins et al. (21) had previously shown that ionophore can induce $T$ cell anergy. The use of general ionophores is not practical for immunotherapy, because calcium fluxes across irritable membranes, including the heart and brain, would be toxic. The HLA-derived peptides, however, appear highly specific. Indeed, as described above, they are well tolerated both in animal models and humans. It appears that the prolonged increase in intracellular calcium in T cells may interfere with the function of numerous intermediates in cell activation. Many molecules, including calcineurin, calcium- and calmodulin-dependent protein $\mathrm{ki}$ nase, and calmodulin, bind calcium (16). The rise in intracellular calcium may interrupt specific pathways, resulting in a negative signal, causing $T$ cell anergy, rather than a positive signal, causing $\mathrm{T}$ cell activation (Fig. 4).

A popular model of $T$ cell activation has resulted from the study of the effects of immunosuppressive drugs, cyclosporine and FK506 (22) (Fig. 5). Both of these potent immunosuppressive drugs bind to intracellular proteins, called immunophilins

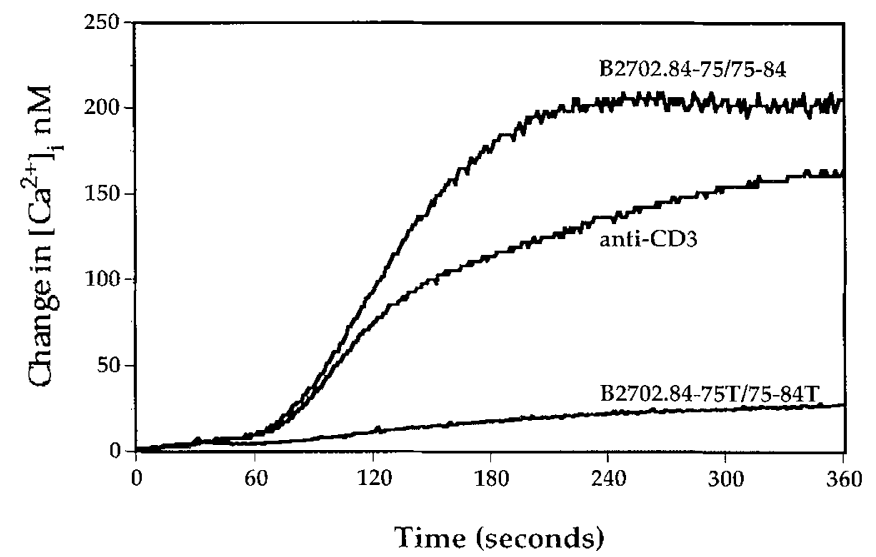

Figure 3. An HLA-B2702.84-75/75-84 inverted dimeric peptide causes an increase in intracellular calcium similar to that seen with anti-CD3 treatment. A control peptide, corresponding to HLA-B2705.84-75/75-84, has no effect. For methods, see Goldberg et al. (16).

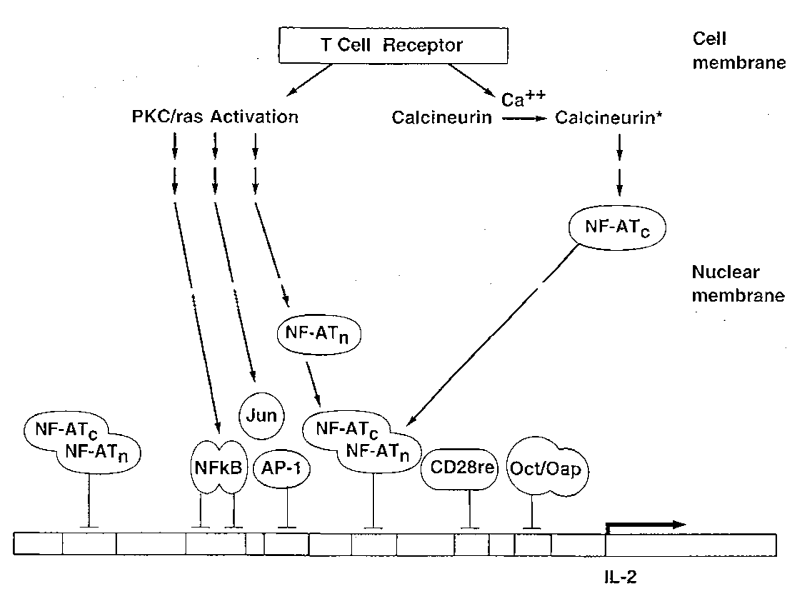

Figure 4. T cell activation. The diagram shows the important pathways involved in $\mathrm{T}$ cell activation. It is possible that the calcium flux associated with HLA peptide binding results in a negative signal causing $T$ cell anergy, rather than a positive signal for $\mathrm{T}$ cell activation (16).
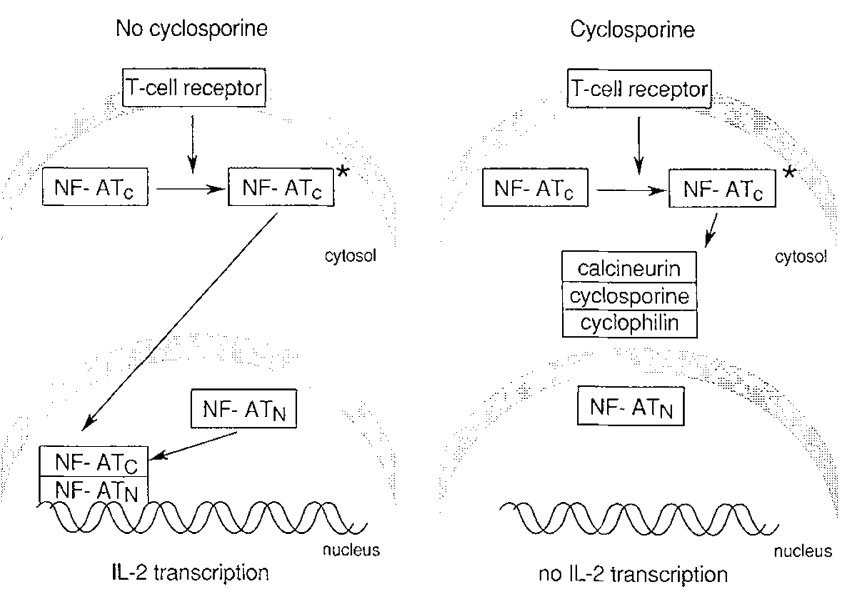

Figure 5. A model of the mechanism of action of cyclosporine. In the presence of cyclosporine, a cytoplasmic component of the nuclear factor of $\mathrm{T}$ cell activation, NF-AT nuclear component, NF-AT , is unable to bind DNA, and there is no IL-2 transcription. Reprinted with permission from Krensky (2).

(23). Cyclosporine binds to cyclophilins and FK506 to FK binding proteins. Once the drug binds to its binding protein(s), the complex is capable of attaching to an important intermediate in signal transduction, calcineurin. This phosphatase appears to regulate the nuclear translocation of NF-AT. NF-AT binding to the IL-2 enhancer is required for IL-2 transcription. Inhibition of this function appears to be a major mechanism of action of these immunosuppressive drugs. The immunomodulatory HLA-derived peptides described above decrease NF-AT binding to the IL-2 enhancer and decrease the function of the NF-AT in reporter gene assays (Goldberg J, Krensky AM, Clayberger $\mathrm{C}$, unpublished observations). Work in progress will define the molecular basis for this observation. It is possible that the calcium flux results in binding of a repressor to the IL-2 promoter, thus locking the $\mathrm{T}$ cell into a state of anergy (Fig. 4).

Members of the hsp70 family bind immunomodulatory HLA derived peptides. The second approach to defining the mechanism of action of the peptides has been to determine the cell receptors which bind them. Fluorescence tagged peptides 
bind to the surface of T lymphocytes (Noessner E, Naftzger C, Clayberger C, Krenshy AM, manuscript submitted). Therefore, $\mathrm{T}$ lymphocytes were radiolabeled and biotinylated peptides were used to isolate binding proteins via streptavidin and run on an SDS-PAGE. This approach has identified two proteins of approximately 72 and $74 \mathrm{kD}$ which bind the peptides. A variety of studies by Noessner et al. prove that these binding proteins are members of the heat shock protein 70 family (manuscript submitted). They are the constitutive hsc70 and heat-inducible hsp 70. Although it is not yet clear by what mechanism hsp 70 binding may result in the functional effects described above, a series of experiments do show tight correlation between functional effects, the calcium flux, and hsp binding. Most convincing is the fact that a single amino acid change in the peptide, an isoleucine for threonine change at residue 80 of the HLA sequence results in a loss of functional effects, change in intracellular calcium, the inhibition of the NF-AT reporter gene assays, and hsp binding (Noessner E, et al., submitted). Recent studies by Fourie et al. (24) show that the Bw4.75-84 peptide precisely matches the expected binding motif for hsp 70 family members and predicts the importance of the isoleucine to threonine change. The central threonine appears to be the major anchor for binding in the hsp70s and the leucines present laterally in the peptide appear to strengthen binding. The alternate hydrophobicity of the peptide is also a major determinant of hsp 70 binding.

\section{SUMMARY}

Thus, we have identified synthetic peptides corresponding to linear sequences of HLA class I molecules which have profound immunomodulatory effects both in vitro and in vivo and are nontoxic in humans (25). While clinical trials are on-going, great progress has been made in understanding the mechanism of action of the peptides. They bind to hsp70 family members, cause prolonged calcium fluxes in T cells, and induce anergy. Although these actions were unexpected, further studies will undoubtedly provide new insights into the mechanisms of $\Upsilon$ cell activation and regulation of the immune response. For example, release of calcium from intracellular stores induced by anti-T cell receptor antibodies is caused by inositol phosphates, whereas that mediated by the HLA peptides is not (16), suggesting additional mechanisms for changes in intracellular calcium. Further studies of the ultimate destination of the NF-AT proteins after anergy induction will be important to understanding differences between the mechanisms of activation versus anergy. Most importantly, however, these peptides appear to be of utility for treatment of a variety of immune mediated diseases. If $\mathrm{T}$ cell anergy can be induced and tolerance maintained, the potential applicability of these peptides for treatment of diverse diseases, such as diabetes, multiple sclerosis, systemic lupus erythematosus, and organ transplant rejection, can be envisioned. And, most importantly, the ability to induce and maintain tolerance may portend a new era of real cures for these chronic, debilitating, and lethal diseases (19).

\section{REFERENCES}

1. Krensky AM, Weiss A, Crabtree G, Davis M, Parham P 1990 Mechanisms of disease: T lymphocyte-antigen interactions in transplant rejection. N Engl J Med 322.510-517

2. Krensky AM 1993 Transplantation Immunobiology' In: Holliday MA, Barratt TM, Avner ED (cds) Pediatric Nephrology, 3rd Ed, Williams \& Wilkins, Baltimore, pp 1373-1389

3. Clayberger C, Rosen M, Parham P, Krensky A 1990 Recognition of an HLA public determinant (Bw4) by human allogeneic cytotoxic T lymphocytes. J Immunol 144:4172-4176

4. Salter RD, Clayberger C, Lomen CE, Krensky AM, Parham P 1987 In vitro mutagenesis at a single residue introduces $B$ and $T$ cell epitopes into a class I HLA molecule. J Exp Med 166:283-288

5. Parham P, Clayberger C, Zorn SL, Ludwig DS, Schoolnik GK, Krensky AM 1987 Inhibition of alloreactive cytotoxic T lymphocytes by peptides from the $\alpha_{2}$ domain of HLA-A2. Nature 325:625-628

6. Clayberger C, Parham P, Rothbard J, Ludwig DS, Schoolnik GK, Krensky AM 1987 HLA-A2 peptides can regulate cytolysis by human allogeneic T lymphocytes. Nature 330:763-765

7. Ways JP, Parham P 1983 The antigenic structure of HLA-A2: An analysis with competitive binding assays and monoclonal antibodies. J Immunol 131:856-863

8. Wesley PK, Clayberger C, Lyu S-c, Krensky AM 1993 The CD8 corcceptor interaction with the $\alpha_{3}$ domain of HLA class I is critical to the differentiation of human cytotoxic T-lymphocytes specific for HLA-A2 and HLA-Cw4. Hum Immunol $36: 149-155$

9. Salter RD, Norment AM, Chen BP, Clayberger C, Krensky AM, Littman DR, and Parham P 1989 Polymorphism in the $\alpha_{3}$ domain of HLA-A molecules affects binding to CD8. Nature 338:345-347

10. Salter RD, Benjamin RJ, Wesley PK, Buxton S, Garrett TPJ, Clayberger C, Krensky AM, Norment AM, Littman DR, Parham P 1990 A binding site for the $\Upsilon$ cell co-receptor, CD8, on the $\alpha_{3}$ domain of HLA-A2. Nature 345:41-46

11. Clayberger C, Lyu S-c, DeKruyff R, Parham P, Krensky AM 1994 Peptides corresponding to the $\mathrm{CD} 8$ and $\mathrm{CD} 4$ binding domains of HLA molecules block T lymphocyte immune responses in vitro. J Immunol 153:946-951

12. Landegren U, Ramstedt U, Axbcrg I, Ulberg M, Jondal M, Wigzell H 1982 Selective inhibition of $\mathrm{T}$ ccll cytotoxicity at levels of target recognition or initiation of lysis by monoclonal OKT3 and Leu-2a antibodies. J Exp Med 155:1579-1584

13. Miccli MC, Parnes JR 1993 Role of CD4 and CD8 in $T$ cell activation and differentiation. Adv Immunol 53:59-122

14. Clayberger C, Lyu S-c, Pouletty P, Krensky AM 1993 Peptides corresponding to $T$ cell receptor-HLA contact regions inhibit class 1 restricted immune responses. Transplant Proc 25:477-8

15. Davis MM, Bjorkman PJ $1988 \mathrm{~T}$ cell antigen receptor genes and antigen recognition. Nature 334:395-402

16. Goldberg JE, Clayberger C, Krensky AM 1995 Tolerance induction by synthetic peptides corresponding to HLA class I sequences. In: Alexander JW (cd) Tolerance Induction. Springer-Verlag, New York (in press)

17. Nisco S, Vriens P, Hoyt G, Lyu S-c, Farfan F, Pouletty P, Krensky AM, Clayberger C 1994 Induction of allograft tolerance in rats by an HLA class I derived peptide and cyclosporine A. J Immunol 152:3786-3792

18. Nickerson PW, Stcurer W, Steiger J, Strom TB 1994 In pursuit of the "holy grail": Allograft tolerance. Kidney Int 45:S40-S49

19. Krensky AM, Clayberger C 1994 Prospects for induction of tolerance in renal transplantation. Pediatr Nephrol 8:772-779

20. Weiss A, Littman DR 1994 Signal transduction by lymphocyte antigen receptors. Cell 76:263-274

21. Jenkins MK, Mueller D, Schwartz RH, Carding S, Bottomley K, Stadecker MJ, Urdahl KB, Norton SD 1991 Induction and maintenance of anergy in mature T cells. Adv Exp Med Biol 292:161-176

22. Crabtree GR, Clipstone NA 1994 Signal transduction betwecn the plasma membrane and nucleus of $T$ lymphocytes. Annu Rev Biochem 63:1045-1083

23. Schreiber SL, Crabtree GR 1992 The mechanism of action of cyclosporin A and FK506. Immunol Today 13:136-142

24. Fourie AM, Sambrook JF, Gething M-JH 1994 Common and divergent peptide binding specificities of hsp70 molecular chaperones. J Biol Chem 269:30470-30478

25. Krensky AM, Clayberger C 1994 The induction of tolerance to alloantigens using HLA based synthetic peptides. Curr Opin Immunol 6:791-796 